\title{
Postoperative pain assessment based on numeric ratings is not the same for patients and professionals: A cross-sectional study
}

\author{
Jacqueline F.M. van Dijk ${ }^{\mathrm{a}, *}$, Albert J.M. van Wijck ${ }^{\mathrm{a}}$, Teus H. Kappen ${ }^{\mathrm{a}}$, Linda M. Peelen ${ }^{\mathrm{a}, \mathrm{b}}$, \\ Cor J. Kalkman ${ }^{a}$, Marieke J. Schuurmans ${ }^{c}$ \\ ${ }^{a}$ University Medical Centre Utrecht, Department of Perioperative Care and Emergency Medicine, The Netherlands \\ ${ }^{\mathrm{b}}$ University Medical Centre Utrecht, Julius Centre for Health Sciences and Primary Care, The Netherlands \\ ${ }^{\mathrm{c}}$ University Medical Centre Utrecht, Department of Nursing Science, The Netherlands
}

\section{A R T I C L E I N F O}

\section{Article history:}

Received 27 December 2010

Received in revised form 7 July 2011

Accepted 16 July 2011

\section{Keywords:}

Analgesics

Guideline

Pain measurement

Postoperative pain

\begin{abstract}
A B S T R A C T
Background: Numeric pain scores have become important in clinical practice to assess postoperative pain and to help develop guidelines for treating pain. Professionals need the patients' pain scores to administer analgesic medication. However, do professionals interpret the pain scores in line with the actual perception of pain by the patients? Objective: The study aim was to assess which Numerical Rating Scale (NRS) pain score was considered bearable on a Verbal Rating Scale (VRS) by patients and professionals. Methods: This prospective study examined the relationship between the Numerical Rating Scale and a Verbal Rating Scale. The patients $(n=10,434)$ rated their pain the day after surgery on the 11 -point NRS ( $0=$ no pain and $10=$ worst imaginable pain $)$ and a VRS comprising five descriptors: "no pain"; "little pain"; "painful but bearable"; "considerable pain"; and "terrible pain". The first three categories together ("no pain", "little pain" and "painful but bearable") were considered "bearable" and the last two categories ("considerable pain" and "terrible pain") were deemed as "unbearable" pain. The professionals $(n=303)$ were asked to relate the numbers of the NRS to the words of the VRS.

Results: Most patients considered NRS 4-6 as "bearable" pain. Among professionals, anesthesiologists, Post Anaesthesia Care nurses, and ward nurses interpreted NRS scores in the same way as the patients. Only the Acute Pain Nurses interpreted the scores differently; they considered NRS of 5 and higher to be not bearable.

Conclusions: Some care providers and patients differ in their interpretation of the postoperative NRS scores. A risk of overtreatment might arise when health care providers rigidly follow guidelines that prescribe strong analgesics for pain scores above 3 or 4 without probing the patient's preference for pharmacological treatment.
\end{abstract}

(c) 2011 Elsevier Ltd. All rights reserved.

\section{What is already known about the topic?}

- Pain assessment is the foundation of pain management when a patient is experiencing postoperative pain. A

\footnotetext{
* Corresponding author at: University Medical Centre Utrecht, Pain Clinic L02.502, Department of Perioperative Care and Emergency Medicine, P.O. Box 85500, 3508 GA Utrecht, The Netherlands.

Tel.: +31 8875 56163; fax: +31 887555511 .

E-mail address: j.f.m.vandijk@umcutrecht.nl (Jacqueline F.M. van Dijk).
}

frequent and thorough assessment of patients' pain by registered nurses provides information to achieve optimal pain relief.

- Clinical guidelines are developed for postoperative pain management based on the patient's pain score. In these guidelines different cut-off points are used to treat the pain.

\section{What this paper adds}

- Patients and professionals do interpret the numeric rating scores for postoperative pain differently. 
- The findings suggest a potential risk of overtreatment if the pain is assessed by the Numeric Rating Scale (NRS) only.

\section{Introduction}

According to the American Pain Society guidelines (APS, 1995) for pain management, postoperative pain should be assessed regularly and documented carefully. The intensity of pain should be evaluated and recorded at intervals depending on the severity of pain and the clinical situation. Pain assessment and management is a significant part of nursing care and the pain is mostly assessed through verbal communication with the patient. The Numeric Rating Scale (NRS) is frequently used for this purpose: the patient is asked to score the pain on an 11 point scale, where 0 indicates no pain and 10 indicates the worst imaginable pain. The NRS is considered a valid and reliable pain assessment tool (Breivik et al., 2000; DeLoach et al., 1998; Good et al., 2001).

The patient's NRS score is a leading indicator in the postoperative pain treatment. Many guidelines for pain management recommend prescription of analgesics on the basis of the patients' NRS pain score (APS, 1995; Gordon et al., 2005; VMS, 2009). However, the NRS threshold for prescribing analgesics varies: some guidelines for acute and cancer pain chose an NRS cut-off >4 (APS, 1995; Gordon et al., 2005) while at least in one other, also for acute and cancer pain, an NRS cut-off $>3$ is the criterion for administering analgesics (VMS, 2009). Furthermore, in clinical practice not all patients with an NRS pain score above the treatment threshold are willing to accept the analgesic treatment offered mostly because they still consider the pain as "bearable". This suggests that professionals and patients might perceive the necessity for pain treatment differently. If so, health care providers who strictly follow current guidelines could be at risk of overtreating some patients.

The aim of the study was to investigate how postoperative NRS pain scores of the patients relate to the presence of "bearable" versus "unbearable" pain. In a prospective study, the postoperative NRS pain scores were compared with the same patients' adjectival descriptions of pain on a Verbal Rating Scale (VRS). The agreement between patients and professionals on the relationship between the NRS and VRS was then studied on the basis of comparisons between the two scales. We hypothesized that patients and professionals might differ in their interpretation of NRS scores.

\section{Methods}

\subsection{Study design}

We describe a cross-sectional study of a large sample of patients admitted for elective surgery. The current study was part of a large cluster-randomized study, implementing a prediction rule for improving the treatment of postoperative nausea and vomiting. In this study, 23,000 in- and out-patients participated. The study was approved by the institutional Ethics Committee of the University
Medical Centre in Utrecht. It was not necessary to obtain informed consent from the patients because pain measurement is part of clinical care. Informed and voluntary consent of the health care professionals was assumed by return of a completed questionnaire.

\subsection{Subjects}

Between March 16th, 2006 and December 21st, 2007, all adult patients scheduled for elective surgery at the University Medical Centre of Utrecht were recruited. The following patients were excluded: those who were transferred directly to an intensive care unit; who needed postoperative ventilatory support; who had complications followed by a second operation; who did not understand the verbal questions of the research nurse; or who underwent ambulatory surgery. All patients received a written brochure preoperatively giving information about postoperative pain measurement and treatment, in accordance with the protocol of the hospital's Acute Pain Service.

Furthermore, we conducted a national survey in which 303 professionals participated: anesthesiologists, Acute Pain Nurses, nurses working on the Post Anaesthesia Care Unit (PACU), student PACU-nurses and ward nurses. The health professionals were a convenience sample. During one week in May, 2008 the PACU nurses and nurses on the surgical wards of the UMC Utrecht were visited and invited to participate in the study. In addition, the nurses in training in the UMC Utrecht for PACU-nurse and working in different hospitals in the Netherlands were invited to participate. The anesthesiologists were randomly selected from a national anaesthesia congress. The Acute Pain Nurses, registered as members of the Dutch association for pain nurses in 2008, were approached by email. All health professionals were personally informed and invited to participate by the pain nurse who was not involved in patient care.

\subsection{Data collection}

Trained research nurses who were not involved in the postoperative care asked the patients about their pain at rest on the day after surgery. The 11 point NRS was used, where 0 indicates no pain and 10 the worst pain imaginable. The VRS used in this study gives five expressions on a scale of increasing burden: "no pain" (VRS 0), "little pain" (VRS 1), "painful but bearable" (VRS 2), "considerable pain" (VRS 3 ) and "terrible pain" (VRS 4). The first three categories together ("no pain", "little pain" and 'painful but bearable') were considered 'bearable' and the last two categories together ('considerable pain' and 'terrible pain') were deemed as 'unbearable' pain. Furthermore, information concerning gender, age, surgical procedure and type of anaesthesia was gathered. The professionals were invited to relate the NRS to the VRS; they received a hand-delivered questionnaire with the five descriptions constituting the aforementioned VRS and were asked to relate the numbers $0-10$ of the NRS to these words. The questionnaires were hand-collected when once completed. No demographic data from the health professionals were collected. 


\subsection{Statistical analyses}

Data were analyzed using descriptive statistics on complete cases. Results for continuous variables were expressed as mean (SD) or as median for variables following normal and non-normal distributions, respectively. Categorical data were expressed as frequencies. Correlations between NRS and VRS were calculated by the Spearman rank correlation coefficient. Statistical testing for non-normally distributed variables used the MannWhitney test, and for categorical values the $\chi^{2}$ test. Sensitivity and specificity of the NRS in detecting 'unbearable' pain were calculated using cut-off points VRS $\leq 2$ (bearable pain) and VRS $>2$ (unbearable pain) and were represented by a Receiver Operator Characteristic (ROC) curve. To analyze the relationship between VRS and NRS scores, we used the modal score (the most frequent value) of the numbers of the NRS per VRS category and examined whether this relationship differed between patients and professionals. To be able to detect differences between males and females and different age groups we did subgroup analyses for gender and age. Statistical analyses were performed using SPSS Statistical Software, version 15.0 (SPSS Inc., Chicago, IL). The results were considered statistically significant if the $p$-values were less than 0.05 .

\section{Results}

\subsection{Patients}

The demographic and perioperative data are presented in Table 1. Data from 10,576 surgical inpatients were eligible for the current study; the other 12,424 patients underwent ambulatory surgery or did not meet the inclusion criteria. An NRS and VRS pain score pair was obtained $24 \mathrm{~h}$ after surgery from 10,434 patients. Data on one or both scales were incomplete for 142 patients, mainly because they were too sick to determine the pain score; failed to understand; were confused; or were uncooperative.

Table 1

Demographic and perioperative data ( $n=10,434$ patients).

\begin{tabular}{lc}
\hline Age, mean \pm SD (range) & $52 \pm 17(18-98)$ \\
Gender, $n(\%)$ & $5348(51)$ \\
Female & $5086(49)$ \\
Male & \\
Type of surgery, $n(\%)$ & $2097(20)$ \\
General & $1988(19)$ \\
ENT/faciomaxillary & $1058(10)$ \\
Orthopedic & $974(9)$ \\
Neurosurgery & $965(9)$ \\
Urology & $868(8)$ \\
Gynecologic & $838(8)$ \\
Plastic surgery & $676(6)$ \\
Vascular surgery & $593(6)$ \\
Eye surgery & $226(2)$ \\
Cardiothoracic & $151(1)$ \\
Other & \\
Type of anaesthesia, $n(\%)$ & $9182(88)$ \\
General & $1252(12)$ \\
Locoregional & \\
\hline
\end{tabular}

ENT: ear, nose and throat surgery.

Values are numbers (\%).

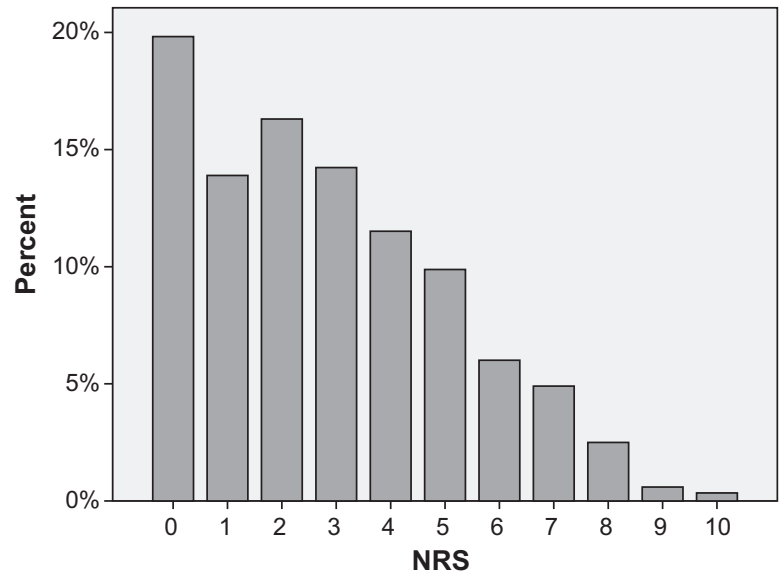

Fig. 1. Pain scores on the Numeric Rating Scale $24 \mathrm{~h}$ after surgery in percentages.

Fig. 1 shows the distribution of the NRS scores of actual pain at rest $24 \mathrm{~h}$ after the operation. The median NRS score was 2. Twenty-four percent of the patients scored an NRS $>4$; this is the threshold value for pain treatment according to various guidelines (APS, 1995; Gordon et al., 2005). In general, women reported higher pain scores than men (median 3 versus 2, respectively; $p<0.001$ ). Older patients (aged 65 and older) reported lower pain scores than younger patients (median 2 versus 3; $p<0.001$ ) (Table 2).

Fig. 2 shows the distribution of the VRS scores of actual pain at rest $24 \mathrm{~h}$ after surgery. Both the median and the mode of the VRS scores were 1 (little pain). In total, $22.7 \%$ of the patients reported 'no pain' (VRS 0), 38.9\% reported 'little pain' (VRS 1), 29.4\% reported 'painful but bearable' (VRS 2), $8.3 \%$ reported 'considerable pain' (VRS 3 ) and $0.7 \%$ reported 'terrible pain' (VRS 4). Women consistently reported more severe pain scores than men $(p<0.001)$. Older patients reported less severe pain scores than younger patients $(p<0.001)$ (Table 3).

\subsection{Professionals}

One hundred and forty anesthesiologists participated (response rate 100\%), along with 50 Acute Pain Nurses (response rate 94\%); 33 PACU nurses (response rate 100\%); 16 nurses in training for PACU-nurse (response rate $100 \%$ ); and 67 nurses on the ward (response rate 100\%).

Table 2

Differences in NRS pain scores.

\begin{tabular}{lll}
\hline & Median pain score & $p$-Value \\
\hline Gender $(n)$ & & \\
$\quad$ Male $(5086)$ & 2 & $<0.001^{\mathrm{a}}$ \\
$\quad$ Female $(5348)$ & 3 & \\
Age $(n)$ & & $<0.001^{\mathrm{a}}$ \\
$\quad \geq 65$ years $(7760)$ & 2 & \\
$\quad<65$ years $(2674)$ & 3 &
\end{tabular}




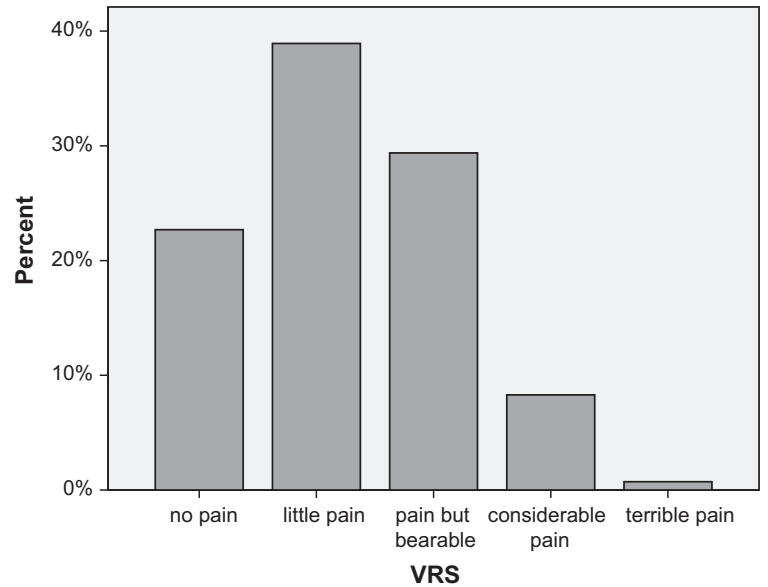

Fig. 2. Pain scores on the Verbal Rating Scale $24 \mathrm{~h}$ after surgery in percentages.

\subsection{The relation of the NRS to the VRS}

The VRS and NRS scores of the patients were significantly correlated (Spearman correlation coefficient $r=0.84, p<0.001)$. Twenty-four percent of the postoperative patients reported an NRS pain score $>4$, while $9 \%$ reported 'considerable' or 'terrible pain' on the VRS. The patients associated NRS 0 with VRS 'no pain'; NRS 1-3 with VRS 'little pain'; NRS 4-5 with VRS 'painful but bearable'; NRS 6-8 with VRS 'considerable pain'; and NRS 9-10 with VRS 'terrible pain'.

As different guidelines show various NRS cut-off points to determine the need for treatment with analgesics, the sensitivity and specificity of the NRS scores and VRS 'bearable' and 'unbearable' were calculated for different NRS cut-off points. Fig. 3 shows an ROC curve depicting the sensitivity and 1 -specificity for these cut-off points. Using an NRS cut-off point of $4,17 \%$ of the patients considered NRS $>4$ to be 'bearable' pain ( 1 - specificity) and $5 \%$ considered it as 'unbearable' pain ( 1 - sensitivity). So using an NRS cut-off value $>4$ for analgesic administration, $17 \%$ of the patients would be incorrectly classified as having unbearable pain, possibly resulting in overtreatment, while $5 \%$ would be undertreated. With a cut-off point of NRS $>3,30 \%$ of the patients would be overtreated and $3 \%$ would be undertreated.

Fig. 4 shows the distribution of the relationship between the NRS and VRS according to the patients and the professionals. The PACU and ward nurses interpreted

Table 3

Differences in VRS pain scores.

\begin{tabular}{lcc}
\hline & Considerable and terrible pain & $p$-Value \\
\hline Gender $(n)$ & & \\
$\quad$ Male $(5086)$ & $7.6 \%$ & $<0.001^{\mathrm{a}}$ \\
$\quad$ Female $(5348)$ & $10.3 \%$ & \\
Age $(n)$ & & \\
$\quad \geq 65$ years $(7760)$ & $8.0 \%$ & $<0.001^{\mathrm{a}}$ \\
$\quad<65$ years $(2674)$ & $9.4 \%$ & \\
\hline a
\end{tabular}

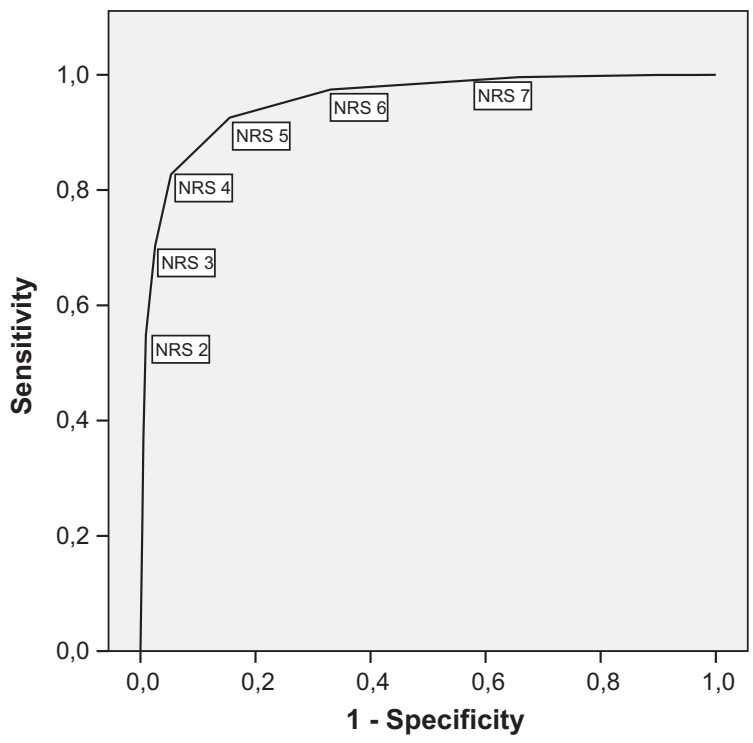

Fig. 3. ROC curve of bearable pain (VRS $\leq 2$ ) and unbearable pain (VRS $>2$ ) with the different NRS cut-off points for 10,434 patients.

the NRS and VRS scores in the same way: NRS 0 equated with VRS 'no pain'; NRS 1-3 with VRS 'little pain'; NRS 4-5 with VRS 'painful but bearable'; NRS 6-8 with VRS 'considerable pain'; and NRS 9-10 with VRS 'terrible pain'. The anesthesiologists interpreted NRS 1 as 'no pain' but their other ratings were identical to those of the PACU and ward nurses. The Acute Pain Nurses interpreted the scores differently: NRS 1-2 'little pain'; 3-4 'painful but bearable'; 5-7 'considerable pain'; and 8-10 'terrible pain'. The distribution of the NRS scores over the VRS categories given by the Acute Pain Nurse was shifted to the left in comparison with those of the other professionals and patients, because they assigned lower NRS scores to the VRS categories.

\section{Discussion}

The present study distinguishes 'bearable' from 'unbearable' postoperative pain and analyzes the relationship between NRS and VRS scores as assessed by postoperative patients and professionals. We found that most patients (65\%) with NRS 4-6 considered their pain bearable. Among the professionals, the anesthesiologists, PACU and ward nurses interpreted the NRS scores in the same way as the patients. Only the Acute Pain Nurses interpreted the scores differently.

In previous studies, different descriptions of pain have frequently been used: no; mild; moderate; and severe pain. In a recent study on pain after orthopedic surgery, the patients related NRS $1-3$ to mild; 4-5 to moderate; and 610 to severe pain (Dihle et al., 2006). After coronary artery bypass grafting, NRS 1-3 was related to mild; 4-6 to moderate; and 7-10 to severe pain (Mendoza et al., 2004). These studies show that in postoperative pain the upper boundary for mild pain is NRS 3 and for moderate pain NRS 5 or 6 . These results are comparable to the findings of the 

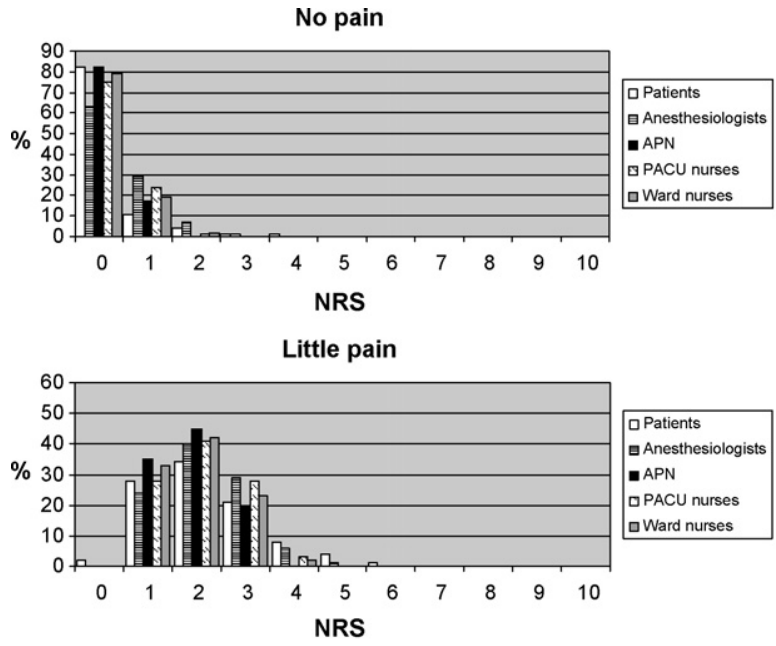

Painful, but bearable

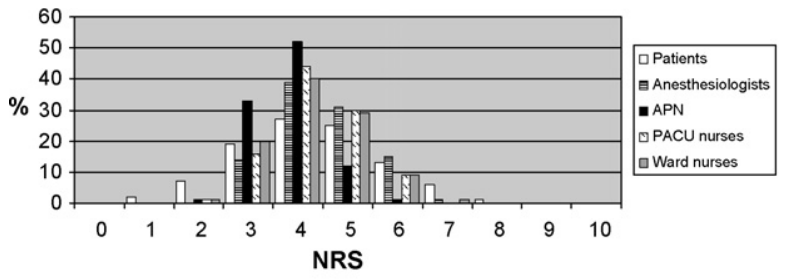

Considerable pain
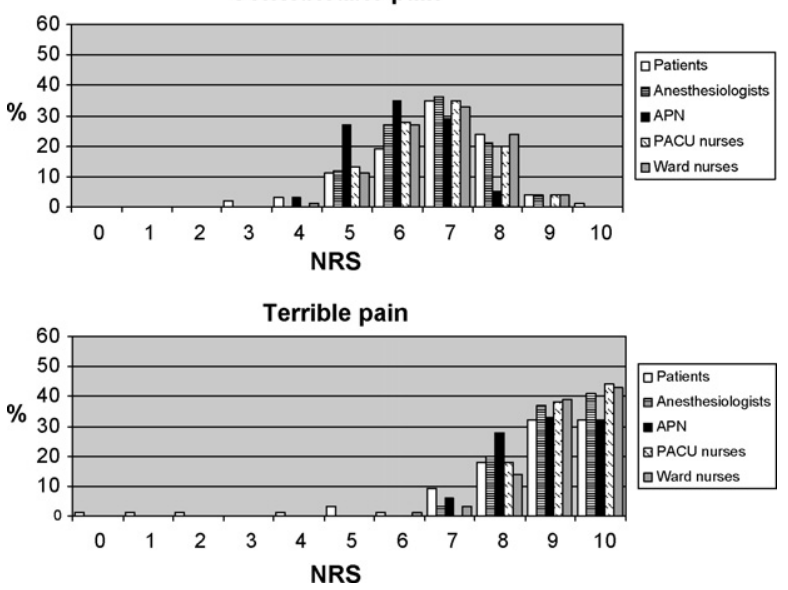

Fig. 4. The relations made of the NRS with the VRS by patients and professionals: Anesthesiologists, Acute Pain Nurses, Post Anaesthesia Care Unit nurses and ward nurses. Spearman correlation coefficient NRSVRS $r=0.84, p<0.001$.

current study. However, instead of moderate pain we used the term 'painful but bearable'. For severe pain we distinguished 'considerable' and 'terrible pain'. Several previous studies have demonstrated that postoperative patients suffer moderate to severe pain despite the use of guidelines (Apfelbaum et al., 2003; Chung and Lui, 2003; Dolin et al., 2002) and that nurses often underestimate the severity of postoperative pain of the patients in their care (Jamison et al., 1997; Taylor et al., 2003; Zelman et al., 2003). It is, however, uncertain to what extent the patients who indicate 'moderate' pain really suffer. In the current study most patients with NRS 4-6 considered their pain as bearable. This relates with other studies where the patients were satisfied with their postoperative pain relief although they still had moderate pain (Apfelbaum et al., 2003; Jamison et al., 1997; Hawkins, 1997). It is possible that most patients experienced moderate but 'bearable' pain and that the nurses did not underestimate the patients' pain.

Most patients with NRS 4-6 considered their pain bearable. Possible reasons are that patients do not understand the NRS pain scores or refuse pain therapy because of side effects or fear for addiction (Wilder-Smith and Schuler, 1992). It is unknown why some patients bear acute pain to some degree and why others do not. Nurses should not only ask the patient about the pain score and follow the guidelines rigidly but also communicate with the patient about the pain and pain therapy (McDonald et al., 2000). The numerical pain ratings are only one dimension of the patient's subjective experience of pain (Hanks, 2008) and adequate pain relief cannot be reliably achieved using opioid analgesics without a high risk of adverse effects (White and Kehlet, 2007).

The results of the current study indicate that the patients and the professionals have a different view of the range of numerical ratings that indicate 'bearable' or 'unbearable' pain.

In particular, Acute Pain Nurses tended to overestimate the severity of pain as perceived by the patient when interpreting NRS scores. Of all health care professionals, the nurses operate most closely to the patient in postoperative care and they can make independent medication decisions for pain relief. The results of this study suggest that the PACU and ward nurses were best informed about what the patients consider as 'bearable' pain. In contrast, the Acute Pain Nurses evaluated the pain scores differently from the patients; they more often overestimate the intensity of pain. In most hospitals the Acute Pain Nurse is the coordinator of the Acute Pain Service and responsible for the in-service education of health care professionals. Although he/she often daily checks up on the patients with Patient Controlled Analgesia or epidural analgesia, the Acute Pain Nurse is not as close to the patient as the ward and PACU nurses are. Another possible explanation of the observed differences is that the Acute Pain Nurses are typically aware of the results of several former studies which have repeatedly demonstrated underassessment and undertreatment of postoperative pain (Idvall et al., 2005; Sloman et al., 2005; Zalon, 1993).

What does 'moderate' pain or an NRS 5 or 6 mean for the patient in order to decide on the need for opioid administration? In the present study, many patients who reported an NRS of 5 or 6 considered their condition to be 'painful but bearable'. A patient who indicates that his/ her pain is 'bearable' might not necessarily want to receive additional analgesic medication, even though all guidelines suggest starting treatment on the basis of these NRS scores. Although we did not ask the patient if he/she would need treatment for his pain, and we did not measure the administered amount of analgesics, the data imply a potential risk of overtreatment when health care providers 
follow the postoperative pain guidelines to the letter. Because a number of studies have demonstrated poor control of the postoperative pain (Apfelbaum et al., 2003; Chung and Lui, 2003; Dolin et al., 2002) the Joint Commission on Accreditation of Health Care Organizations defined new standards for pain management in their standards manual (Philips, 2000). In addition, the American Pain Society and the Dutch VMS recently provided recommendations for improving the quality of acute and cancer pain management (Gordon et al., 2005; VMS, 2009). In these recommendations increasing emphasis is placed on reducing the patients' pain scores, which entails increased analgesic usage. Decreasing the intensity of pain is a laudable objective and one of the pillars of health care. In postoperative pain management, both undertreatment and overtreatment are undesirable. Unrelieved pain has adverse psychological and physiological consequences e.g., more complications and prolonged hospital stay (Watt-Watson et al., 1999). On the other hand, improved pain control should not jeopardize patient safety (Taylor et al., 2003). Unnecessary use of analgesics, especially opioids, increases the patient's discomfort because of the side effects, such as nausea, vomiting and pruritus. Unnecessary opioid administration also increases the probability of sedation and potentially dangerous respiratory depression (Cashman and Dolin, 2004; Taylor et al., 2005). An increase of opioid oversedation is reported after the implementation of the new standards of the Joint Commission (Vila et al., 2005). Therefore, to balance the risks of overtreatment and undertreatment in this range of numerical pain scores, the health care provider might simply add the following question: "would you like to have an analgesic?" In addition, the patients' level of consciousness should be assessed when patients receive (additional) opioids.

\subsection{Limitations}

The present study is subject to a number of limitations. We measured the postoperative pain scores of the patient in rest. These pain scores can be different of the pain scores at movement. Nevertheless, the purpose of the study was to examine what numeric ratings were bearable to the patient. The health care professionals participated in this study were a convenience sample and no demographic data were collected. Regarding their level of experience, however, given the fact that all professionals working in the shift in which the data were collected participated, the sample was representative. Another potential source of error is the order in which the two pain scores were asked. All patients first indicated the severity of their pain on the VRS and then on the NRS. We chose this order because we anticipated that patients might try to 'convert back' to an adjective in the VRS once they had given an NRS score (which requires mental conversion from a subjective feeling to a number). Although in theory it can make a difference whether NRS or VRS is asked first, a recent study reported that error rates were not related to the order of presentation of the scales (Gagliese et al., 2005).

The data we used for this study were part of a larger study implementing a prediction rule to improve the treatment of postoperative nausea and vomiting (PONV). We are aware of the relationship between PONV and pain. In the larger study, the anesthesiologists were more focused on PONV and possibly prescribed less analgesia. However, we assume that there was no interference from the PONV study, because we studied two scales for expressing the severity of pain, and not the severity of the pain itself. This assumption was verified by repeating our analyses with data from the control group of the PONV study only; this yielded similar results.

\section{Conclusions}

By combining data from a large sample of surgical patients with a national survey of health care workers, a lack of agreement is found between the patients and the Acute Pain Nurses on what constitutes 'bearable' pain in relation to the reported NRS scores. The Acute Pain Nurses tended to overestimate the severity of pain when the patients reported intermediate NRS scores, whereas most patients considered NRS 4-6 to be 'bearable' pain. These findings suggest a potential risk of overtreatment, if the pain is assessed by the NRS only and treatment decisions are based solely on a patient's NRS response. Specifically asking a patient whether the pain is bearable and he/she would like to receive additional analgesics might circumvent this problem.

Conflict of interest. None of the authors has any potential conflicting interest in this study.

Funding. This study is supported by ZonMW (Clinical Trial nr. NCT00293618), the Netherlands Organization for Health Research and Development. The study is supported by the Division of Perioperative Care and Emergency Medicine, University Medical Centre Utrecht, The Netherlands.

Ethical approval. The study was approved by the institutional Ethics Committee of the University Medical Centre in Utrecht.

\section{References}

American Pain Society, 1995. Quality improvement guidelines for the treatment of acute pain and cancer pain. JAMA 274, 1874-1880.

Apfelbaum, J.L., Chen, C., Mehta, S.S., Gan, T.J., 2003. Postoperative pain experience: results from a national survey suggest postoperative pain continues to be undermanaged. Anesthesia and Analgesia 97, 534-540.

Breivik, E.K., Björnsson, G.A., Skovlund, E., 2000. A comparison of pain rating scales by sampling from clinical trial data. Clinical Journal of Pain 16, 22-28.

Cashman, J.N., Dolin, S.J., 2004. Respiratory and haemodynamic effects of acute postoperative pain management: evidence from published data. British Journal of Anaesthesia 93 (2), 212-223.

Chung, J.W., Lui, J.C., 2003. Postoperative pain management: study of patients' level of pain and satisfaction with health care providers' responsiveness to their reports of pain. Nursing and Health Sciences 5 , 13-21.

DeLoach, L.J., Higgins, M.S., Caplan, A.B., Stiff, J.L., 1998. The Visual Analogue Scale in the immediate postoperative period: intrasubject variability and correlation with a Numeric Scale. Anesthesia and Analgesia 86, 102-106.

Dihle, A., Helseth, S., Paul, S.M., Miaskowski, C., 2006. The exploration of the establishment of cutpoints to categorize the severity of acute postoperative pain. Clinical Journal of Pain 22, 617-624. 
Dolin, S.J., Cashman, J.N., Bland, J.M., 2002. Effectiveness of acute postoperative pain management: I. Evidence from published data. British Journal of Anaesthesia 89, 409-423.

Good, M., Stiller, C., Zausziewski, J.A., Cranston Anderson, G., Stanton-Hicks, M., Grass, J.A., 2001. Sensation and distress of pain scales: reliability, validity and sensitivity. Journal of Nursing Measurement 3, 219-238.

Gordon, D.B., Dahl, J.L., Miaskowski, C., McCarberg, B., Todd, K.H., Paice, J.A., Lipman, A.G., Bookbinder, M., Sanders, S.H., Turk, D.C., Carr, D.B., 2005. American Pain Society recommendations for improving the quality of acute and cancer pain management. Archives of Internal Medicine 165, 1574-1580.

Gagliese, L., Weizblit, N., Ellis, W., Chan, V.W., 2005. The measurement of postoperative pain: a comparison of intensity scales in younger and older surgical patients. Pain 117, 412-420.

Hanks, S., 2008. The law of unintended consequences, when pain management leads to medication errors. Pharmacy and Therapeutics 33, 420-425.

Hawkins, R.M., 1997. The role of the patient in the management of post surgical pain. Psychology and Health 12, 565-577.

Idvall, E., Berg, K., Unosson, M., Brudin, L., 2005. Differences between nurse and patient assessments on postoperative pain management in two hospitals. Journal of Evaluation in Clinical Practice 11, 444-451.

Jamison, R.N., Ross, M.J., Hoopman, P.R., Griffin, F.R., Levy, J.R., Daly, M.R., Schaffer, J.L., 1997. Assessment of postoperative pain management: patient satisfaction and perceived helpfulness. Clinical Journal of Pain 13, 229-236.

McDonald, D.D., McNulty, J., Erickson, K., Weiskopf, C., 2000. Communicating pain and pain management needs after surgery. Applied Nursing Research 13, 70-75.

Mendoza, T.R., Chen, C., Brugger, A., Hubbard, R., Snabes, M., Palmer, S.N., Zhang, Q., Cleeland, C.S., 2004. Lessons learned from a multiple-dose postoperative analgesic trail. Pain 109, 103-109.
Philips, D.M., 2000. JCAHO pain management standards are unveiled. JAMA 284, 428-429.

Sloman, R., Rosen, G., Rom, M., Shir, Y., 2005. Nurses' assessment of pain in surgical patients. Journal of Advanced Nursing 52, 125-132.

Taylor, S., Voytovich, A.E., Kozol, R.A., 2003. Has the pendulum swung too far in postoperative pain control? American Journal of Surgery 186, 472-475.

Taylor, S., Kirton, O.C., Staff, I., Kozol, R.A., 2005. Postoperative day one: a high risk period for respiratory events. American Journal of Surgery 190, 752-756.

Vila, H., Smith, R.A., Augustyniak, M.J., Nagi, P.A., Soto, R.G., Ross, T.W., Cantor, A.B., Strickland, J.M., Miguel, R.V., 2005. The efficacy and safety of pain management before and after implementation of hospital wide pain management standards: is patient safety compromised by treatment based solely on numerical pain ratings? Anesthesia and Analgesia 101, 474-480.

VMS veiligheidsprogramma, 2009. Vroege herkenning en behandeling van pijn. Den Haag, The Netherlands.

Watt-Watson, J.H., Clark, A.J., Finley, G.A., Watson, C.P., 1999. Canadian pain society position statement on pain relief. Pain Research and Management 4, 75-78.

White, P.F., Kehlet, H., 2007. Improving pain management: Are we jumping from the frying pan into the fire? International Anaesthesia Research Society 105, 10-12.

Wilder-Smith, C.H., Schuler, L., 1992. Postoperative analgesia: pain by choice? The influence of patient attitudes and patient education. Pain 50, 257-262.

Zalon, M.L., 1993. Nurses' assessment of postoperative patients' pain. Pain $54,329-334$.

Zelman, D.C., Hoffman, D.L., Seifeldin, R., Dukes, E.M., 2003. Development of a metric for a day of manageable pain control: derivation of pain severity cut-points for low back pain and osteoarthritis. Pain 106, 35-42. 